\title{
Palomena prasina [Hemiptera: Pentatomidae] vibratory signals and their tuning with plant substrates
}

\section{Research Article}

Jernej Polajnar ${ }^{1,2, *}$, Andreja Kavčič ${ }^{1}$, Alenka Žunič Kosi ${ }^{1}$, Andrej Čokl ${ }^{1}$

${ }^{1}$ National Institute of Biology,

SI-1000 Ljubljana, Slovenia

${ }^{2}$ Fondazione Edmund Mach,

IT-38010 S. Michele all'Adige, Italy

Received 31 January 2013; Accepted 19 March 2013

Abstract: Palomena prasina is interesting for the study of vibrational communication within the Pentatomid subfamily Pentatominae, because its host range is limited to woody plants, unlike the better known Nezara viridula, whose vibrational communication is commonly used as a model for the whole family. The vibrational repertoire of $P$. prasina was described several decades ago and is redescribed in this paper using modern methods for non-contact vibration recording. Additionally, we hypothesized that this species has retained the capacity for signal frequency variation necessary for tuning to resonance properties of various host plants of Pentatominae, but if the signals are emited in the absence of mechanical feedback, they are tuned more specifically to their native acoustic environment - woody plants. By recording live bugs signalling on different substrates and comparing spectral properties of their signals among substrates, we found that there is a match between the signals emitted on a woody branch and those emitted on a non-resonant surface, while spectral properties of signals emitted on herbaceous plants differ. Our findings provide evidence in support of the signal tuning hypothesis and shed further light on the crucial role of substrate in vibrational communication of insects.

Keywords: Pentatominae • Vibrational communication • Signal propagation • Frequency characteristics

(C) Versita Sp. z 0.0 .

\section{Introduction}

Palomena prasina (Linnaeus, 1761) (Heteroptera: Pentatomidae) is a univoltine herbivorous species of the subfamily Pentatominae, the stink bugs, that feed on various deciduous trees and shrubs. It has a Palearctic distribution and is common in Europe where it has status as a minor pest of apple, pear, raspberry and hazelnut, particularly in those orchards that are situated in the vicinity of broadleaved woodlands [1]. One notable exception to this minority status is in Turkey, where the species is the dominant insect pest in hazelnut production [2]. Vibrational repertoire of $P$. prasina was first described several decades ago by its air-borne sound component, which was registered with a conventional microphone and amplified [3]. The authors described the female and male calling songs, and two types of male courtship songs, assuming that the "calling song" is a type of song used for long-range detection and localization of mates, while "courtship song" consisting of more elaborate signals is used for precise species recognition analogous with Nezara viridula [3]. The airborne components' spectral composition was similar to all described vibratory signals in the subfamily Pentatominae to date $[4,5]$, but no correlation with properties of the substrate was made at the time.

In recent years, the importance of substrate for vibrational communication has come into focus. Physical features of the substrate determine various signal transmission properties, such as propagation velocity, frequency filtering, and resonance [6]. Plant tissues are generally considered low-pass filters [5-9], imposing the upper frequency limit of signals used for efficient long-range communication in Pentatomine stink bugs. The dominant frequencies of all described songs produced in Pentatomine stink bugs by abdomen 
vibration to date fall in the range of $70-150 \mathrm{~Hz}$ with higher harmonics extending up to $600 \mathrm{~Hz}$ [5]. Resonance properties of sedge plants (Cyperus alternifolius L.) are one known exception that would in principle enable efficient transmission of vibration with frequencies up to $500 \mathrm{~Hz}$ or more [10]. However, this is probably due to the relatively simple geometry of sedge plants which have long straight stems. The resonant properties of substrates with more complex geometry effectively limit frequency of long-range signals to $200 \mathrm{~Hz}$ or lower for insects of this size (J. Polajnar, unpublished data), although stronger signallers such as the large tree wetas (Orthoptera: Stenopelmatidae) can effectively produce long-range plant-borne signals at frequencies of several $\mathrm{kHz}$ [11].

Palomena prasina is interesting for the study of vibrational communication within the family Pentatomidae, because its host range appears to be limited to woody plants, unlike the better known $N$. viridula, whose vibrational communication is commonly used as a model for the whole family [12]. Nezara viridula is extremely polyphagous and not limited to any type of plant substrate. It feeds, mates and oviposits on various herbaceous and woody plants, depending on the season and geographical location $[13,14]$. In contrast with $N$. viridula, $P$. prasina is expected to have adapted to transmission properties of its specialized host range. Such a phenomenon has been convincingly demonstrated in treehoppers (Hemiptera: Membracidae) where spectral composition of signals is further determined by the species' choice of micro-habitat [15], although not in terms of individual plasticity. The difference in signal transmission properties between herbaceous and woody host plants is likely due to differences in tissue stiffness, which influences propagation velocity, and therefore changing resonant properties [6].

Assuming that the bugs are able to detect mechanical feedback from the substrate and respond by adjusting signalling properties (mainly frequency), they are in turn expected to adjust those properties to values at which transmission is as efficient as possible. If one or more resonant peaks of a particular type of substrate fall within the range of signal variability, each individual can tune its signals to its current acoustic environment. Otherwise, resonant properties may impose constraints on communication. Data from several related and sympatric species show that the dominant frequency of signals can vary by a factor of 2 [4], which is reflected by the receiver's preference [16]. Individual animals can also rapidly respond to changes in their acoustic environment, such as onset of noise, by modifying the frequency of their vibratory emission during a signalling bout [17]. We hypothesized that the more specialized, but closely related $P$. prasina has retained the capacity for frequency variation, but that the animals' signalling behavior has adapted ("tuned") to their native acoustic environment. The study reported here had two main goals: first, we endeavoured to reinvestigate vibrational communication of $P$. prasina using non-contact optical methods to detect the substrate-borne component of their emissions, and to enable comparison with the other members of this subfamily in which vibrational repertoire has been described more recently. The second goal was to compare signal tuning with different substrates. Properties of the host plant of this species differ from the common bean or sedge plants that have been frequently used in the study of stink bug vibrational communication. According to our hypothesis, the spectral composition of signals recorded on a nonresonant loudspeaker membrane would match the spectral composition of signals recorded on a hazel branch, while on other substrates, it would differ because of different transmission properties of those substrates.

\section{Experimental Procedures}

Palomena prasina specimens for experiments were collected in the surroundings of Ljubljana (Brod) and at the Slovenian coast, at the end of September 2009 and in subsequent years. The insects were kept separated by sex in plastic cups at room temperature and 16:8 day:night period. Insects were reared on a diet of fresh beans (Phaseolus vulgaris L.), sunflower seeds (Hellianthus annus L.) and raw peanuts (Arachis hypogaea L.).

All experiments were conducted in the laboratories of the Department of Entomology, National Institute of Biology, Ljubljana, from 8 A.M. until 5 P.M. The following types of substrates were used: membrane of a mid-range cone loudspeaker, and three plant species: common bean ( $P$. vulgaris), sedge (Cyperus alternifolius L.) and common hazel (Corylus avellana L.). Of those, four different bean plants, one sedge stem with leaf rosette and two hazel branches were used. Bean and sedge plants were grown in pots with a mix of soil and vermiculite, while the hazel branches were picked from the wild and fixed using cotton wool at the mouth of a plastic Erlenmeyer flask, which was filled with water. Small $\left(\sim 0.25 \mathrm{~cm}^{2}\right)$ pieces of reflective foil were glued to different locations on each plant, such as the middle of the leaf and around the stem to ensure good laser reflection. In the case of the loudspeaker, reflective foil was glued to the center.

The dimensions of each plant are presented in Figure 1 and Table 1. The loudspeaker and pots with 

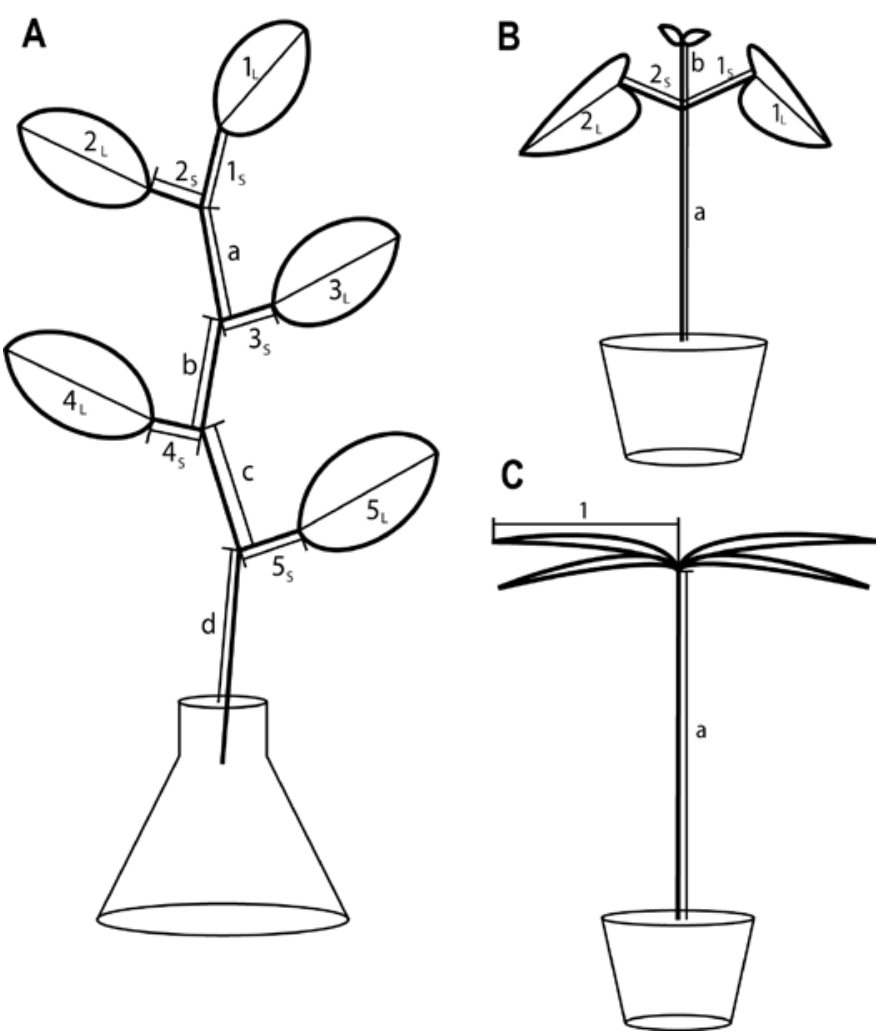

Figure 1. Geometry of the plants used for recording. Measurements are given in Table 1. A: common hazel (Corylus avellana), B: common bean (Phaseolus vulgaris), C: sedge (Cyperus alternifolius).

plants were placed on a vibration proof table to reduce environmental noise. Strictly speaking, loudspeakers as finite physical objects also exhibit resonance, but the unmounted cone speaker used in our experiments had a nearly flat response to excitation with varying frequencies in the relevant frequency range (J. Polajnar, unpublished data), unlike plant substrates [10], so we considered it non-resonant for this purpose.

One or more bugs were placed on a substrate and left to walk on it freely in each trial, except on the loudspeaker where only a single bug was recorded at a time, constrained within the circumference of the membrane by a transparent plastic cover. Vibratory emissions were detected as surface velocity of the plant substrate using a laser-doppler vibrometer (Type PDV 100, Polytec, Waldbronn, Germany). The laser's voltage output was recorded with a computer sound card (Sound Blaster X-Fi, Creative Labs, Milpitas, CA) at $44,100 / \mathrm{s}$ sample rate and stored in an uncompressed wave format for later analysis. The approximate location of the signalling insect was noted, which served as a basis for establishing three classes of distance between the recording point and the emitter for each signal: $0-5 \mathrm{~cm}, 5-15 \mathrm{~cm}$ and $>15 \mathrm{~cm}$. Signals in different distance classes were compared in order to evaluate possible changes due to transmission along the substrate.

\subsection{Terminology and data analysis}

Recordings of vibratory signals were analyzed using Raven Pro 1.4 software [18]. Terminology of temporal characteristics of signals and signal sequences follows [19]: pulse is a discrete unit of sound within a series of similar units, a pulse train is a group of pulses with a characteristic form, and a song is a group of pulses or pulse trains of the same type. Repetition time is the time between the start of two sequential pulses and pause is the time between the end of a pulse and the beginning of the following pulse. Spectral composition of signals was analyzed using Fast Fourier Transform (FFT) computation (Hann window, DFT size $=16.384$ samples) on whole pulses where possible, or pulse trains where single pulses were too short for FFT analysis with these parameters. Dominant frequency is defined as the frequency of the highest peak in a signal's frequency spectrum excluding low-frequency noise below $40 \mathrm{~Hz}$.

The frequency tuning hypothesis was tested with spectral analysis of signals emitted on different substrates. We compared dominant frequency components detected manually within individual spectra, and qualitatively the 


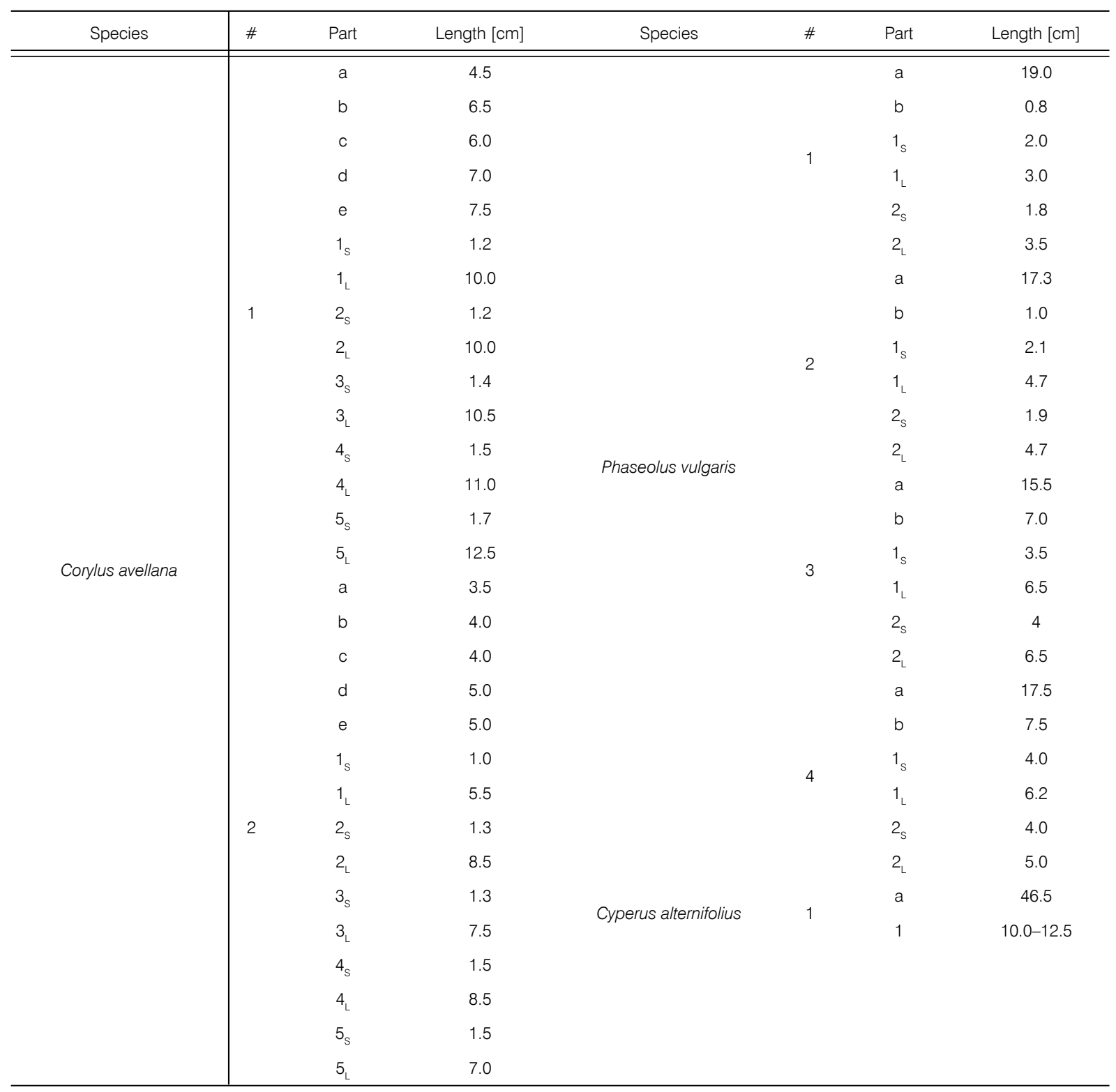

Table 1. Dimensions of plants used for recording. Two common hazel (Corylus avellana) plants, four common bean (Phaseolus vulgaris) plants, and one sedge (Cyperus alternifolius) plant were used.

whole spectra. To compare whole frequency spectra, raw spectrum samples were exported in text format using the Raven Pro 1.4 export function. Then, spectra from all pulses emitted on the same substrate and comparable distance from the recording point were pooled together and normalized to the same maximum amplitude. Finally, averaging of amplitudes was performed across equivalent frequency components of pooled spectra. With the given FFT parameters, each frequency component represents the middle of a $2.7 \mathrm{~Hz}$-wide frequency bin. The averaged spectrum is displayed as average \pm standard deviation for each frequency component. Averaging was performed using a custom-written script in the programming language $\mathrm{R}$ [20].

\section{Results}

\subsection{Vibrational repertoire of $P$. prasina}

We recorded the emission of vibratory signals in 146 mixed-gender trials on a natural substrate. Both males and females readily emitted vibrational signals without special stimulation on all kinds of substrate used, and regardless of the time of recording. We identified one 
A
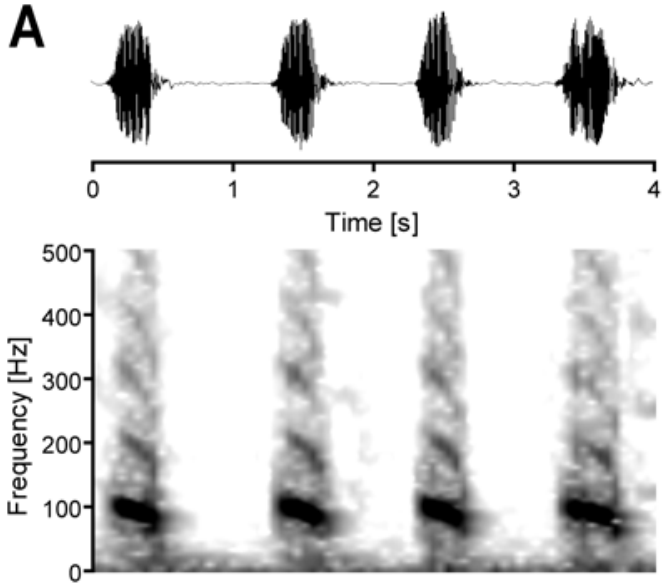

B

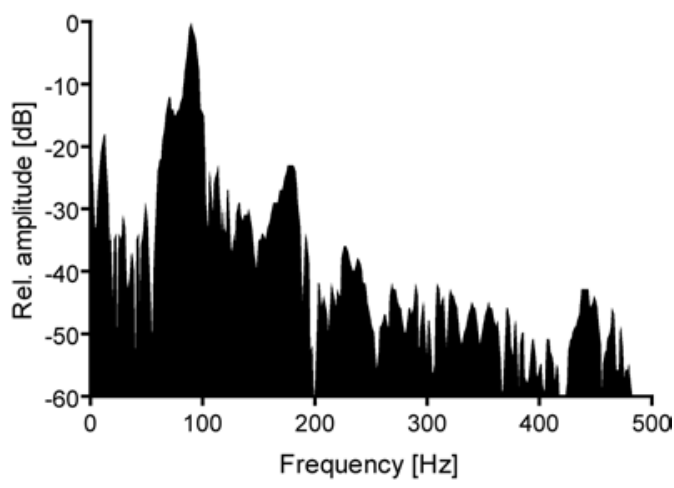

Figure 2. Temporal and spectral properties of an FS pulse train. A: above oscillogram, below spectrogram, B: frequency spectrum of one pulse.

A

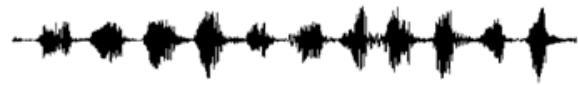

$$
0
$$
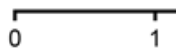

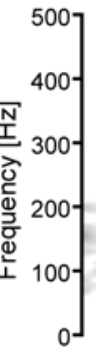

Time [s]
B

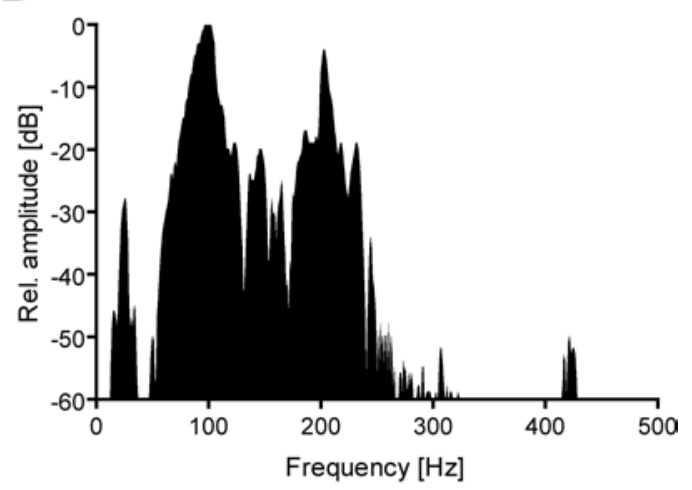

Figure 3. Temporal and spectral properties of an MS-1 pulse train. A: above oscillogram, below spectrogram, B: frequency spectrum of a pair of pulses.

A

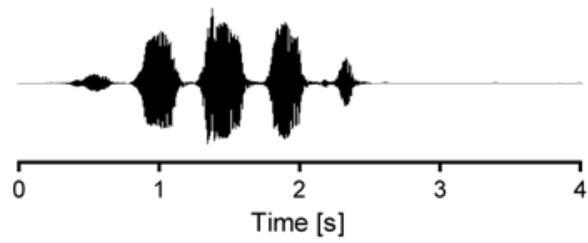

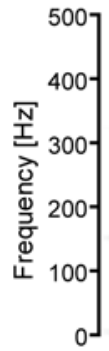

B

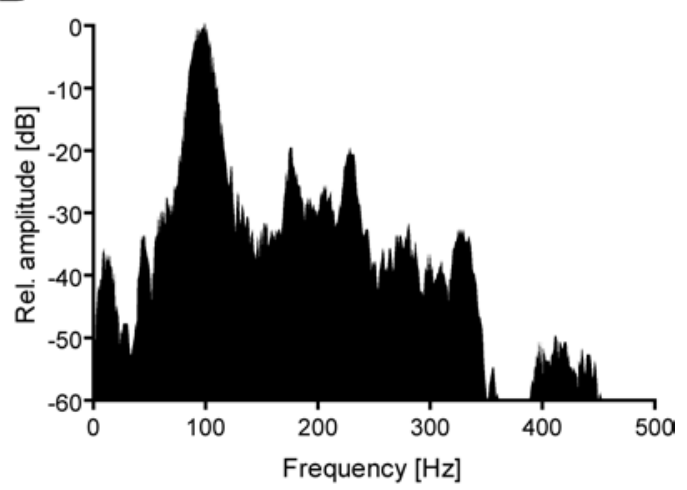

Figure 4. Temporal and spectral properties of an MS-2 pulse train. A: above oscillogram, below spectrogram. B: frequency spectrum of the whole train. 
A

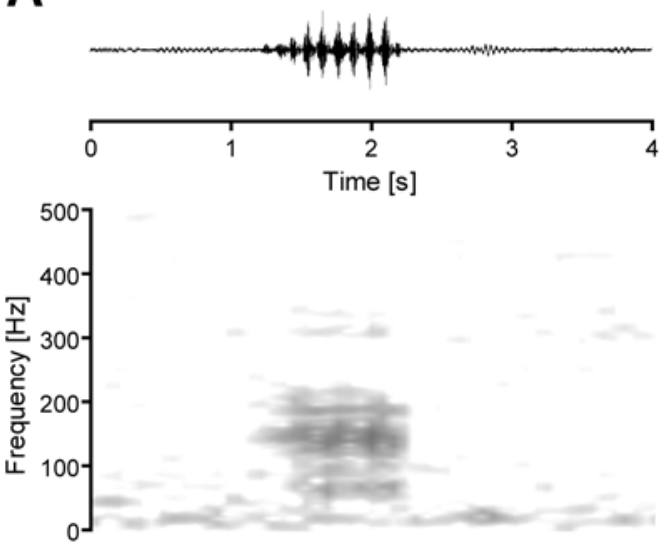

B

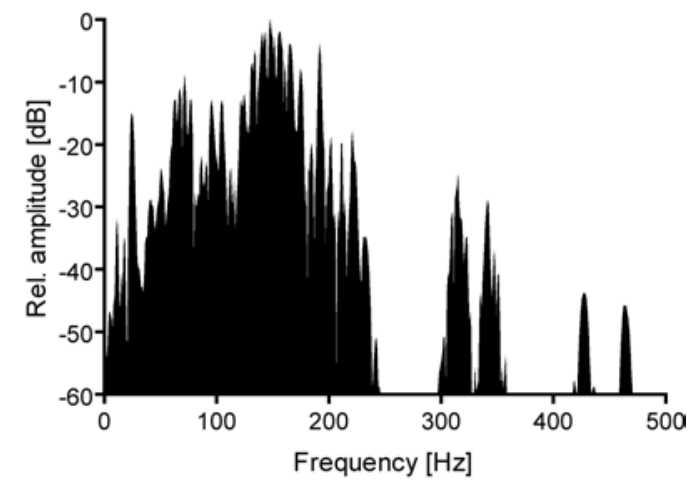

Figure 5. Temporal and spectral properties of an MS-3 pulse train. A: above oscillogram, below spectrogram. B: frequency spectrum of the whole train.

\begin{tabular}{|c|c|c|c|c|c|c|c|c|}
\hline Signal type & Duration [ms] & $\mathrm{N}$ & Repetition time [ms] & N & Dominant frequency $[\mathrm{Hz}]$ & N & No. pulses & N \\
\hline \multicolumn{9}{|c|}{ FS-1 } \\
\hline pulse & $398(217-630)$ & 321 & $1010(268-2476)$ & 556 & $90.4 \pm 3.5$ & 16 & / & l \\
\hline \multicolumn{9}{|c|}{ MS-1 } \\
\hline pulse & $196(101-369)$ & 683 & $311(152-680)$ & 606 & $95.1 \pm 8.1$ & 194 & / & l \\
\hline \multicolumn{9}{|c|}{ MS-2 } \\
\hline first pulse & $413(109-1173)$ & 199 & l & l & / & / & / & l \\
\hline other pulses & $268(87-825)$ & 1005 & 369 (159-999) & 811 & l & / & / & l \\
\hline train & $1689(557-23445)$ & 419 & highly variable & l & $95.5 \pm 7.6$ & 109 & $5(1-93)$ & 419 \\
\hline \multicolumn{9}{|c|}{ MS-3 } \\
\hline pulse & approx. $100^{a}$ & 292 & approx. $100^{a}$ & l & l & l & / & l \\
\hline train & 840 (374-1991) & 302 & highly variable & l & $110-160^{b}$ & 44 & $9(3-24)$ & 292 \\
\hline
\end{tabular}

Table 2. Basic properties of songs emitted by Palomena prasina. Values represent pooled data from all distance classes on the two hazel branches, except dominant frequency of MS-2 trains, where only the data from the distance class closest to the emitter were used. Most values are presented as medians with range in parentheses, except for dominant frequency where average \pm SD is given.

aan estimate obtained by dividing train duration with the number of pulses

ba rough approximation

female (FS) and three male song types (MS-1, MS-2, and MS-3) (Figures 2-5, Table 2). No rival or copulatory vibratory signals were detected, despite extensive recording of multiple pairs on the same plant. Distribution of temporal parameters of all song types was skewed towards lower values, so variability is presented with values for minimum, maximum and median, and nonparametric tests are used for analysis, mostly MannWhitney $U$ test (MW). Temporal parameters presented here are gathered from recordings on all substrates, while spectral parameters were analyzed in recordings on hazel only since we hypothesized that substrates differ in this regard.
The female song (FS) (Figure 2) was the only type of female vibratory emission recorded. It was composed of approximately $0.4 \mathrm{~s}$ pulses repeated every second (Table 2). Sequences of pulses began or ended gradually, often overlapping with male vibratory emissions, so it was usually not possible to determine exact range of pulse numbers or sequence durations. Sequences were, however, very variable and could consist of one or two pulses or up to dozens of pulses emitted in a continuous sequence. The average dominant frequency of pulses was $90.4 \pm 3.5 \mathrm{~Hz}(\mathrm{~N}=16, \mathrm{n}=2)$. Frequency did not change significantly with distance from the emitter (ANOVA; $F(1,14)=5.70, P=0.46)$, so pooled data is presented. 
Male song 1 (MS-1) (Figure 3) was usually emitted by males as a sequence of pulses similar to FS, but with shorter pulse duration and pause (Table 2). Like in the female song, it was not possible to determine exact range of pulse numbers or sequence durations, because sequences began or ended gradually, often overlapping with other animals' vibratory emissions. Frequency analysis was performed on pairs of adjacent pulses due to short duration of individual pulses. The average dominant frequency was $95.1 \pm 8.1 \mathrm{~Hz}(\mathrm{~N}=194, \mathrm{n}=9)$. Frequency did not change significantly with distance from the emitter (ANOVA; $F(2,191)=2.49, P=0.09$ ), so pooled data is presented.

Male song 2 (MS-2) (Figure 4) was emitted in pulse trains with a well defined beginning and end. Number of pulses comprising a train varied by two orders of magnitude, but was usually around five. Consequently, their overall duration also varied by the same extent (Table 2). MS-2 trains were superficially similar to short MS-1 trains, but differed in several aspects. The first pulse of a train was noticeably prolonged compared to the rest and was, on average, significantly longer than other pulses (MW; $U=153645, P<0.001$ ). A single pulse was occasionally emitted alone by a male. Such a pulse appeared similar to other first pulses within MS-2 trains so it was counted as an MS-2 train composed of one pulse. All first pulses within MS-2 trains were ignored for comparison of temporal parameters with MS-1 song. MS-2 pulses were significantly longer (MW; $U=154620$, $\mathrm{P}<0.001)$, with longer pulse repetition time than MS-1 (MW; $U=146877, P<0.001$ ). However, pauses were shorter than MS-1 (MW; $U=134850, P<0.001)$, or sometimes even absent, so the pulses tended to fuse together. Due to short duration of individual pulses, frequency analysis was performed on whole pulse trains. The dominant frequency was in the same range as other songs described above. Frequency did not change significantly with distance from the emitter (ANOVA; $F(2,244)=2.49, P=0.09$ ), so pooled data is presented. Slight downward modulation of frequency was also apparent;dominant frequency decreased approximately $10 \%$ from the beginning to the end of each pulse.

Male song 3 (MS-3) (Figure 5) differed substantially from the previous two song types. It consisted of trains of short, fused pulses with an abrupt beginning and end, usually composed of less than 10 pulses with approximately the same duration throughout (Table 2). It was not unambigously discernible where every individual pulse began or ended, but dividing each train's duration with its number of pulses gives around $100 \mathrm{~ms}$ duration. Peak amplitude was much lower than in MS-2, with the sound energy distributed over a wide frequency range without a clear dominant frequency. Overall, the strongest frequency component was in the range between 110 and $160 \mathrm{~Hz}$. In almost half of the cases (44\%), an MS-3 train was followed immediately by an MS-2 train. Additionally, $7 \%$ of MS-3 trains were followed by an MS-2 train less than two seconds afterwards, and the rest of MS-3 trains were emitted alone, occasionally in roughly regular sequences of trains.

\subsection{Influence of substrate on spectral properties}

We focused on MS-1 for the analysis of the influence of substrate, because that song type was the only one recorded on every kind of substrate with a large enough sample size. Spectrum averaging proved useful for comparing signal types on the frequency axis even though normalization changed amplitude ratios between spectra. Averaged spectra of all signals emitted in the same distance class, and on the same substrate, retained the sharp dominant frequency peak. The dominant frequency of MS-1 signals emitted more than $15 \mathrm{~cm}$ away from the recording point on either of the two hazel branches did not differ significantly (MW; $\mathrm{U}=888, \mathrm{P}=0.35$ ). The peak of the first higher harmonic frequency was more variable. Consequently, it was lower and wider than the dominant frequency peak on the averaged spectrum which was not always the case in individual spectra.

We tested our hypothesis of $P$. prasina's ability for tuning signals to the native woody substrate by comparing dominant frequency of signals emitted on different plants to that of signals emitted on a nonresonant surface, i.e. the loudspeaker membrane. Recordings from all distance classes on each plant were used for analysis. Not all datasets were normally distributed, so the data are presented as medians with ranges (Table 3), and non-parametric tests are used for comparisons. There was a significant difference in dominant frequency between substrates (Kruskal-Wallis test; $\left.X^{2}=126.1, d f=3, P<0.001\right)$. Pairwise comparisons were made between frequency of signals emitted on the loudspeaker membrane as the non-resonant substrate, and those emitted on various natural substrates. Comparison of MS-1 average spectrum between the loudspeaker and a hazel branch (Figure 6A) revealed

\begin{tabular}{c|cc}
\hline Substrate & $\begin{array}{c}\text { Dominant frequency (median and } \\
\text { range) }[\mathrm{Hz}]\end{array}$ & $\mathrm{N}$ \\
\hline \hline Loudspeaker & $95.7(82-115.2)$ & 208 \\
Hazel & $94.8(72.6-126.3)$ & 194 \\
Bean & $98.1(53.8-142.4)$ & 280 \\
Sedge & $110.2(102.1-126.3)$ & 63 \\
\hline
\end{tabular}

Table 3. Dominant frequency of signals emitted on different substrates. 


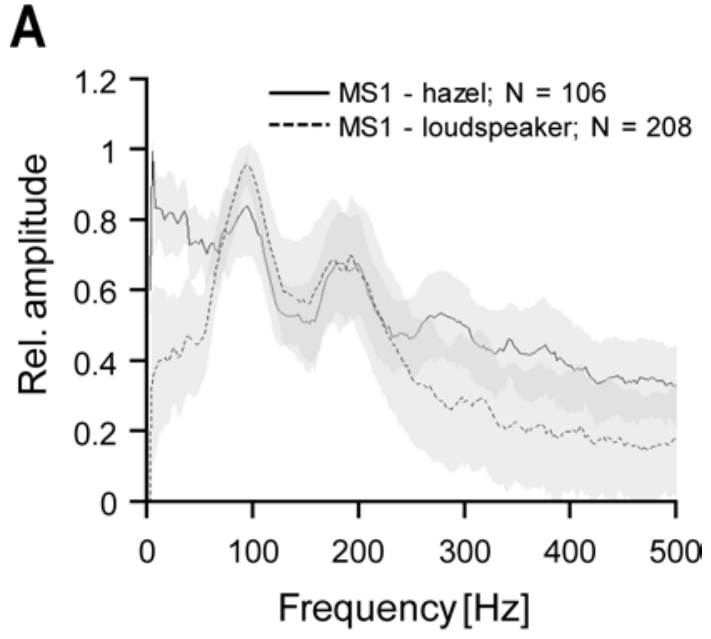

B
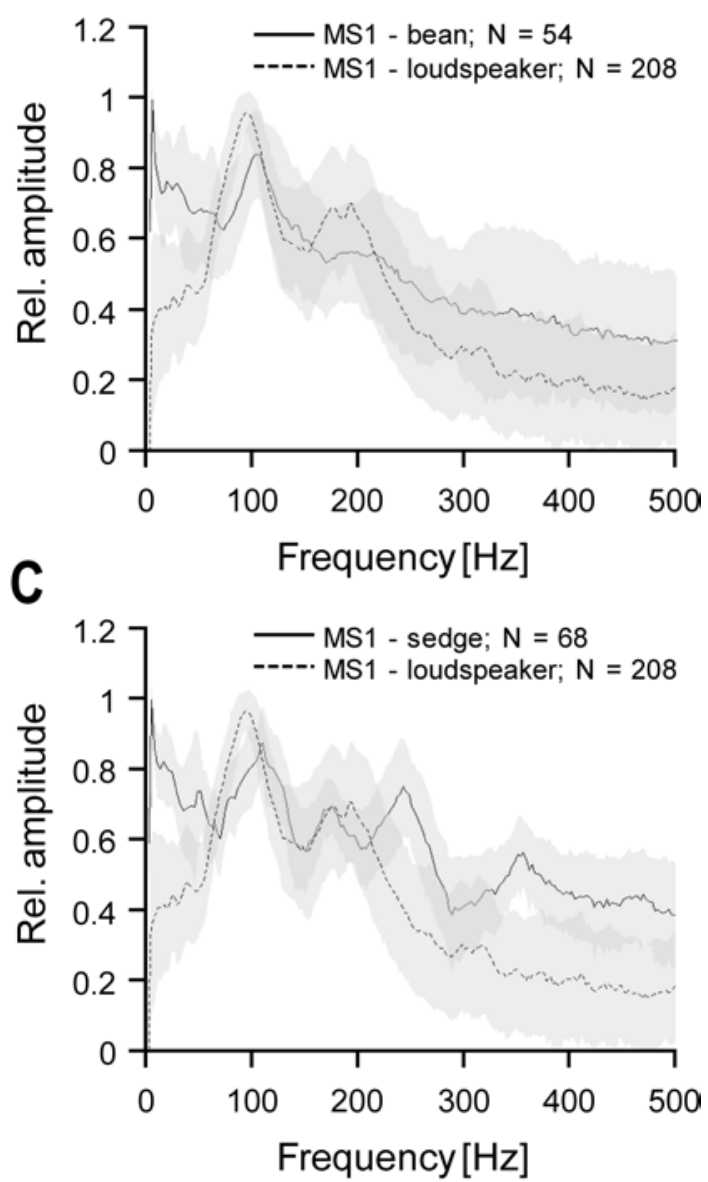

Figure 6. Influence of substrate on spectral properties. Averaged spectra of signals emitted on different substrates are compared to averaged spectrum of signals emitted on a non-resonant substrate (loudspeaker). A: comparison between loudspeaker and hazel; B: comparison between loudspeaker and bean; C: comparison between loudspeaker and sedge. Grey areas represent \pm one standard deviation from the average for each frequency component. virtually no qualitative difference in the dominant and the first harmonic peak, apart from changed amplitude ratios. Dominant frequency itself was the same (MW; $U=19827$, $\mathrm{P}=0.76)$. On the contrary, MS-1 signals emitted on bean plants had significantly higher dominant frequency (MW; $\mathrm{U}=25077, \mathrm{P}<0.01$ ) than on the loudspeaker, which is clearly apparent from the averaged spectra (Figure 6B). Another prominent feature of the averaged spectrum on bean plants is a reduced first harmonic peak compared to the averaged spectrum on loudspeaker. The dominant frequency was also higher on a sedge plant than on the loudspeaker (MW; U=277, P<0.001). Several additional harmonic frequency peaks could be discerned on sedge, ranging upwards to $500 \mathrm{~Hz}$ (Figure 6C). On all plant substrates, the drop-off above $200 \mathrm{~Hz}$ was much less prominent than on a loudspeaker.

The part of the spectrum below the dominant frequency (low-frequency part) was also influenced by the type of substrate. We separated pulses emitted on a hazel branch in low and high background noise conditions for this part of the analysis. We only used signals that clearly fit into either category (i.e. low or high noise) by having signal-to-noise ratio over $20 \mathrm{~dB}$ (low noise) or under $0 \mathrm{~dB}$ (high noise). The noise was caused mainly by the test subjects walking around.

Regardless of the amount of background noise, the low-frequency part of the signals' spectrum was weaker when emitted on the loudspeaker membrane than when emitted on a plant (Figure 6). The lowest frequency component of signals registered on a plant was always stronger than the dominant frequency peak of an averaged spectrum, while on a loudspeaker, there was a constant drop-off below the dominant peak. Dominant frequency of MS1 signals was not influenced by the amount of background noise (MW; $U=195, P=0.15$ ), nor was the rest of the spectrum qualitatively (Figure 7). Other than at the lowest frequency component, the part of the spectrum below the dominant peak was mostly featureless, with the exception of sedge where two prominent resonant peaks were observed.

\section{Discussion}

Sexual communication in $P$. prasina is similar to that described in other species of the stink bug subfamily Pentatominae, with distinct types of vibrational signals, i.e. songs, emitted by both males and females [4]. However, due to the low number of observed matings, we cannot present a detailed overview of the behavioral sequence that leads to copulation in this paper.

Vibrational communication did not appear to be affected by the substrate; both males and females 


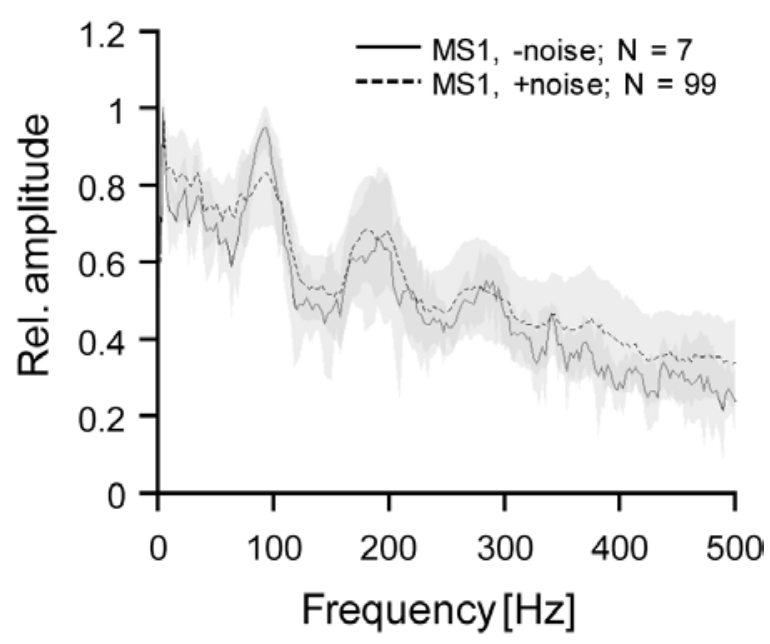

Figure 7. Influence of noise. MS-1 signals emitted in conditions of low background noise (solid line) and high background noise (dashed line) on a hazel branch, recorded $>15$ $\mathrm{cm}$ from the emitter. Grey areas represent \pm one standard deviation from the average for each frequency component.

signalled readily without any external trigger. The recorded song types are the same as already described [3], with one notable exception. A variant of the MS-2 song type was described previously as "a male courtship song with a short preceeding chirp". In our study we found that it is actually a signal composed of two song types, i.e. MS-2 and MS-3. The latter was often followed directly by the former, but MS-3 also was emitted alone, therefore supporting a distinction. We decided not to name male song types more specificly, because it was not possible to discern their behavioral context; either type could be emitted spontaneously, with or without the male switching to other signal types in the same trial. The MS-3 song type is interesting because of its distinct form, which is unlike any currently described song type in Pentatomid bugs except the congeneric Palomena viridissima [3].

A case could still be made for labelling MS-1 as calling song and MS-2 as courtship song. In most other species of Pentatominae, such as Nezara viridula [21], Acrosternum impicticorne, Euschistus heros, Thyanta perditor [22], Thyanta pallidovirens [23], Holcostethus strictus [24], and Edessa meditabunda [25], the song type emitted by males in the calling phase also consists of regularly repeated pulses with a simple amplitude envelope (similar to the MS-1), while close-range courtship in later phases involves more elaborate signals with extensive amplitude modulation (similar to the MS-2), which is assumed to convey species specificity $[5,26]$. However, this is not a strict rule since an opposite phenomenon was described in Acrosternum hilare males [19].
Frequency analysis of signals emitted on different substrates confirmed our hypothesis about signal tuning to the native substrate of this species. The difference in dominant frequency between native and non-native substrates, although small, was highly significant, with the insects' 'default' frequency (as measured on a non-resonant substrate) matching the frequency on a native substrate. The findings suggest a crucial role of the substrate in determining efficient signal frequency, which is supported by other recent research on this subject (For example, see [10,15,27]).

The most obvious possible explanation is a difference in resonance properties of different substrates. In principle, it is energetically most efficient for the signaller to emit signals close to one of the resonance frequencies of the stem, which could represent the proximal mechanism for frequency matching through a simple sensory feedback loop, i.e. detecting amplitude of own signals with given effort and at a range of frequencies. Such a match is suggested by results of simultaneous recording of vibrations on the back of a singing $N$. viridula and the plant substrate, which revealed no difference in dominant frequency between recording points [27]. Neither that study nor the present one revealed any change in dominant frequency with distance from the emitter, which indicates that filtering properties of the plant do not cause dominant frequency change. On the other hand, subdominant peaks varied in both frequency and relative amplitude, which was also the case in the present study.

Biological relevance of the observed match between signaling frequency on non-resonant substrate and the native substrate must lie in the improved efficiency of signal transmission in an environment where a $10 \%$ frequency change can result in 10-30 dB change of amplitude due to attenuation [6]. Frequency specificity of the receptor apparatus is not likely to play a role because Pentatomid bugs have broadly tuned vibrational receptors of several classes that cover the whole range of dominant frequencies observed in this study [5]. The interpretation still relies on several assumptions that need to be tested. Most notably, no studies have so far tried to correlate resonant properties of particular plants to the signal characteristics of the particular animal signalling through them, which would be the logical next step.

There is a significant difference in male signal frequency between treehopers in the Echenopa binotata species complex (Hemiptera: Membracidae) that occur on different host plant species [28]. The difference in this case is in the range of several hundred $\mathrm{Hz}$ and is likely to be a result of micro-habitat choice that determines the most efficient signal frequency [15], as well as divergent 
evolution due to shaping of female preference $[29,30]$. However, this not the adaptive phenotypic plasticity that is implied by our results. A direct effect of environment on the level of individuals has been observed in jumping spiders of the species Habronattus dossenus (Araneae: Salticidae) that occur on widely different substrates, e.g. sand, rocks and leaf litter, where only leaf litter transmits this species' courtship signals effectively [31]. Interestingly, males did not modify their behavior when confined to a suboptimal substrate and simply achieved far lower copulation success with the same effort. Additionally, no spectral difference was found between mating signals of Chrysoperla downesi (Neuroptera: Chrysopidae) recorded on a grass stalk and a conifer branch [32], however, the method used was unlikely to detect differences because the substrate was vibrated artificially with a play-back device that drove the substrate at a predetermined frequency. Only a live signalling animal is relevant for direct comparisons, being possibly able to detect and respond to mechanical feedback of the substrate. Our study is, to our knowledge, the first

\section{References}

[1] Alford D.V., Pests of fruit crops: a color handbook, Academic Press, Burlington, MA, 2007

[2] Tuncer C., Saruhan I., Akça I.., The insect pest problem affecting hazelnut kernel quality in Turkey, Acta Hortic., 2005, 686, 367-375

[3] Čokl A., Gogala A., Blaževič A., Principles of sound recognition in three Pentatomide bug species (Heteroptera), Biol. Vestn., 1978, 26, 81-94

[4] Čokl A., Virant-Doberlet M., Communication with substrate-borne signals in small plant-dwelling insects, Ann. Rev. Entomol., 2003, 48, 29-50

[5] Čokl A., Stink bug interaction with host plants during communication, J. Insect Physiol., 2008, 54, 1113-1124

[6] Michelsen A., Flemming F., Gogala M., Traue D., Plants as transmission channels for insect vibrational songs, Behav. Ecol. Sociobiol., 1982, 11, 269-281

[7] Magal C., Schöller M., Tautz J., Casas J., The role of leaf structure in vibration propagation, J. Acoust. Soc. Am., 2000, 108, 2412-2418

[8] Čokl A., Plants as medium for communication with mechanical vibrations, In: Sapri R.C. (Ed.), Mechanical vibrations: measurement, effects and control, Nova Science Publishers, inc., New York, NY, 2009

[9] Čokl A., Žunič A., Virant-Doberlet M., Predatory bug Picromerus bidens communicates at different frequency levels, Cent. Eur. J. Biol, 2011, 6, 431-439 observation of the dependence of signals on substrate properties directly, on an individual scale. Evolutionary processes were not the focus of this study, although we assume that natural selection is behind the observed phenomenon since signalling behavior must be shaped by the physical properties of acoustic environment [33-35]. Instead, we demonstrated the mismatch between spectral properties of signals emitted on native and those emitted on non-native substrates, where the 'default', as emitted on a non-resonant substrate, matches the spectral properties of signals emitted on a native substrate. Still, the underlying frequency plasticity could in principle enable the population to make host shifts without much detrimental effect to mating success because of the influence of the substrate.

\section{Acknowledgements}

This work was financially supported by the Slovenian Research Agency (Research Programme P1-0255).

[10] Polajnar J., Svenšek D., Čokl A., Resonance in herbaceous plant stems as a factor in vibrational communication of pentatomid bugs (Heteroptera: Pentatomidae), J. R. Soc. Interface, 2012, 9, 18981907

[11] McVean A., Field L.H., Communication by substratum vibration in the New Zealand tree weta, Hemideina femorata (Stenopelmatidae: Orthoptera), J. Zool., Lond., 1996, 239, 101-122

[12] Virant-Doberlet M., Čokl A., Vibrational Communication in Insects, Neotrop. Entomol., 2004, 33, 121-134

[13] Todd J.W., Ecology and behavior of Nezara viridula, Ann. Rev. Entomol., 1989, 34, 273-292

[14] Panizzi A.R., McPherson J.E., James D.G., Javahery M., McPherson R.M., Stink bugs (Pentatomidae), In: Schaefer C.W., Panizzi A.R. (Eds.), Heteroptera of economic importance, CRC Press, Boca Raton, FL, 2000

[15] McNett G.D., Cocroft R.B., Host shifts favor vibrational signal divergence in Enchenopa binotata treehoppers, Behav. Ecol., 2008, 16, 650656

[16] Žunič A., Virant-Doberlet M., Čokl A., Species recognition during substrate-borne communication in Nezara viridula (L.) (Pentatomidae: Heteroptera), J. Insect Behav., 2011, 24, 468-487

[17] Polajnar J., Čokl A., The effect of vibratory disturbance on sexual behaviour of the southern 
green stink bug Nezara viridula (Heteroptera, Pentatomidae), Centr. Eur. J. Biol., 2008, 3, 189197

[18] Charif R.A., Waack A.M., Strickman L.M., Raven Pro 1.4 User's Manual, Cornell Lab of Ornithology, Ithaca, NY, 2010

[19] Čokl A., McBrien H.L., Millar J.G., Comparison of substrate-borne vibrational signals of two stink bug species, Acrosternum hilare and Nezara viridula (Heteroptera: Pentatomidae), Ann. Ent. Soc. Am., 2001, 94, 471-479

[20] R Development Core Team, R: A language and environment for statistical computing, R Foundation for Statistical Computing, Vienna, Austria, 2011

[21] Čokl A., Virant-Doberlet M., Stritih N., Structure and function of songs emitted by southern green stink bugs from Brazil, Florida, Italy and Slovenia, Physiol. Entomol., 2000, 25, 196-205

[22] Blassioli Moraes M.C., Laumann R.A., Čokl A., Borges M., Vibratory signals of four Neotropical stink bug species, Physiol. Entomol, 2005, 30, 175188

[23] McBrien H.L., Čokl A., Millar J.G., Comparison of substrate-borne vibrational signals of two congeneric stink bug species, Thyanta pallidovirens and T. custator accera (Heteroptera: Pentatomidae), J. Ins. Behav., 2002, 15, 715-738

[24] Pavlovčič P., Čokl A., Songs of Holcostethus strictus (Fabricius): a different repertoire among landbugs (Heteroptera: Pentatomidae), Behav. Proc., 2001, 53, 65-73

[25] Cavalcante A., Silva C., Laumann R.A., Cavalcante Ferreira J.B., Blassioli Moraes M.C., Borges M., et al., Reproductive biology, mating behavior, and vibratory communication of the BrownWinged Stink Bug, Edessa meditabunda (Fabr.) (Heteroptera: Pentatomidae), Psyche, 2012, 2012, 1-9

[26] Gogala M., Vibratory signals produced by Heteroptera - Pentatomomorpha and
Cimicomorpha, In: Drosopoulos S., Claridge M.F. (Eds.), Insect sounds and communication, CRC Press, Boca Raton, FL, 2006

[27] Čokl A., Zorović M., Žunič A., Virant-Doberlet M., Tuning of host plants with vibratory songs of Nezara viridula L (Heteroptera: Pentatomidae), J. Exp. Biol., 2005, 208, 1481-1488

[28] Cocroft R.B., Rodríguez R.L., Hunt R.E., Host shifts and signal divergence: mating signals covary with host use in a complex of specialized plant-feeding insects, Biol. J. Linn. Soc. 2012, 99, 60-72

[29] Rodríguez R.L., Ramaswamy K., Cocroft R.B., Evidence that female preferences have shaped signal evolution in a clade of specialized plantfeeding insects, Proc. R. Soc. B, 2006, 273, 25852593

[30] Sullivan-Beckers L., Cocroft R.B., The importance of female choice, male-male competition, and signal transmission as causes of selection on male mating signals, Evolution, 2010, 64, 3158-3171

[31] Elias D.O., Mason A.C., Hoy R.R., The effect of substrate on the efficacy of seismic courtship signal transmission in the jumping spider Habronattus dossenus (Araneae: Salticidae), J. Exp. Biol., 2004, 207, 4105-4110

[32] Henry C.S., Martínez Wells M.L., Adaptation or random change? The evolutionary response of songs to substrate properties in lacewings (Neuroptera: Chrysopidae: Chrysoperla), Anim. Behav., 2004, 68, 879-895

[33] Cocroft R.B., Rodríguez R.L., The Behavioral Ecology of Insect Vibrational Communication, BioScience, 2005, 55, 323-334

[34] Endler J.A., Signals, signal conditions, and the direction of evolution, Am. Nat., 1992, 139, S125-S153

[35] Endler J.A., Some general comments on the evolution and design of animal communication systems, Phil. Trans. R. Soc. B, 1993, 340, 215225 